Article

\title{
Classification of HCV NS5B Polymerase Inhibitors Using Support Vector Machine
}

\author{
Maolin Wang ${ }^{1}$, Kai Wang ${ }^{1}$, Aixia Yan ${ }^{1, *}$ and Changyuan Yu ${ }^{2, *}$
}

1 State Key Laboratory of Chemical Resource Engineering, Department of Pharmaceutical Engineering, Beijing University of Chemical Technology, 15 Bei San Huan East Road, P.O. Box 53, Beijing 100029, China; E-Mails: wangm11240@163.com (M.W.); wangkai0112006@163.com (K.W.)

2 College of Life Science and Technology, Beijing University of Chemical Technology, 15 Bei San Huan East Road, P.O. Box 53, Beijing 100029, China

* Authors to whom correspondence should be addressed; E-Mails: aixia_yan@yahoo.com or yanax@mail.buct.edu.cn (A.Y.); yucy@mail.buct.edu.cn (C.Y.); Tel.: +86-10-6444-7540 (A.Y.); +86-10-6442-1335 (C.Y.); Fax: +86-10-6441-6428 (A.Y.); +86-10-6441-6428 (C.Y.).

Received: 25 November 2011; in revised form: 3 February 2012 / Accepted: 19 March 2012 / Published: 27 March 2012

\begin{abstract}
Using a support vector machine (SVM), three classification models were built to predict whether a compound is an active or weakly active inhibitor based on a dataset of 386 hepatitis C virus (HCV) NS5B polymerase NNIs (non-nucleoside analogue inhibitors) fitting into the pocket of the NNI III binding site. For each molecule, global descriptors, 2D and 3D property autocorrelation descriptors were calculated from the program ADRIANA.Code. Three models were developed with the combination of different types of descriptors. Model 2 based on 16 global and 2D autocorrelation descriptors gave the highest prediction accuracy of $88.24 \%$ and MCC (Matthews correlation coefficient) of 0.789 on test set. Model 1 based on 13 global descriptors showed the highest prediction accuracy of $86.25 \%$ and $\mathrm{MCC}$ of 0.732 on external test set (including 80 compounds). Some molecular properties such as molecular shape descriptors (InertiaZ, InertiaX and Span), number of rotatable bonds (NRotBond), water solubility (LogS), and hydrogen bonding related descriptors performed important roles in the interactions between the ligand and NS5B polymerase.
\end{abstract}


Keywords: hepatitis C virus (HCV); NS5B polymerase inhibitor; classification models; support vector machine (SVM)

\section{Introduction}

Hepatitis $\mathrm{C}$ virus (HCV) infection constitutes a global health problem, which affects more than 170 million individuals [1]. According to WHO figures, over $2 \%$ of the world population is chronically infected with HCV [2]. Hepatitis $\mathrm{C}$ virus (HCV) is a (+)-stranded RNA virus belonging to the Flaviviridae family of enveloped viruses. Previously, the only approved therapy against HCV was pegylated interferon IFNR (IFN), either as monotherapy or in combination with ribavirin. However, this therapy is poorly tolerated and of limited efficacy [3]. The new standard of care (SOC) after recent protease inhibitor approval involves the combination of a protease inhibitor with pegylated-interferon and the oral nucleoside antiviral agent ribavirin [4].

$\mathrm{HCV}$ has six major genotype classes, with genotypes 1 and 2 being most prevalent in the United States, Europe, and Japan. Currently combination drug treatment of genotype 2 or 3 is more successful than treatment of genotype 1 infection [5]. HCV is an enveloped single strand RNA virus and encodes a polyprotein chain of about 3000 amino acids, which is processed into structural and non-structural (NS) proteins [6]. Polyprotein processing by viral and cellular host factors results in four structural proteins (Core, E1, E2, p7) and six nonstructural (NS2, NS3, NS4A, NS4B, NS5A, NS5B) proteins.

NS5B is a RNA-dependent RNA polymerase (RdRp) at the core of the HCV replicative complex. As an RNA-dependant RNA polymerase, it has no counterpart in mammalian cells and so it is expected that inhibition of the enzyme will not cause target-related side effects [7]. Given the essential role of this enzyme in viral replication, it is anticipated that agents capable of disrupting its function will prove efficacious in the treatment of $\mathrm{HCV}$ infections [8]. Due to its necessary role in viral replication, NS5B RNA-dependent RNA polymerase (RdRp) has been one of the most studied viral protein targets for small molecule HCV therapy [9]. Accordingly, several different structural scaffolds of HCV NS5B inhibitors have been identified and many pharmaceutical companies are competing to identify new drugs. ANA-598, ABT-072 and ABT-333 (the molecular structures of these compounds are not disclosed) have been demonstrated efficacy in phase-II clinical trials [7]. Three specific classes of inhibitors that target the polymerase have been reported. These include nucleoside analogue inhibitors (NIs), non-nucleoside analogue inhibitors (NNIs) and pyrophosphate (PPi) analogues [10].

NNIs (non-nucleoside analogue inhibitors) provide an alternative mechanism to target viral polymerases. Greater variability is possible with $\mathrm{HCV}$ inhibitors as multiple allosteric binding sites are present on the HCV polymerase. Up to now, four binding sites of NS5B polymerase have been identified: NNI site I and site II are located in the thumb domain, while III and IV are closer to the active site in the palm domain [10]. In this work, we will work on NS5B NNI III binding site inhibitors. The representative molecules at this binding site are shown in Figure 1. 
Figure 1. Some representative molecular structures of HCV NS5B NNI III binding site inhibitors.

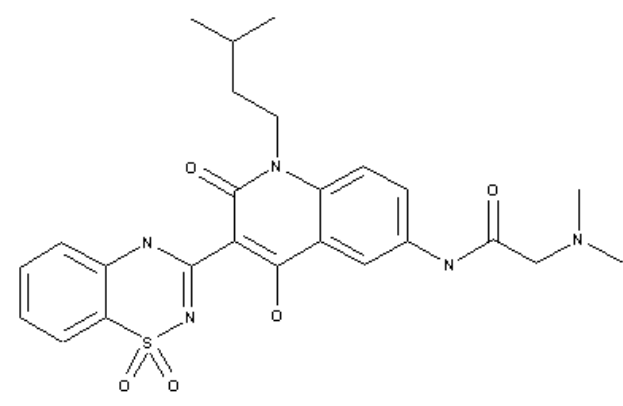

benzothiodiazine analogues

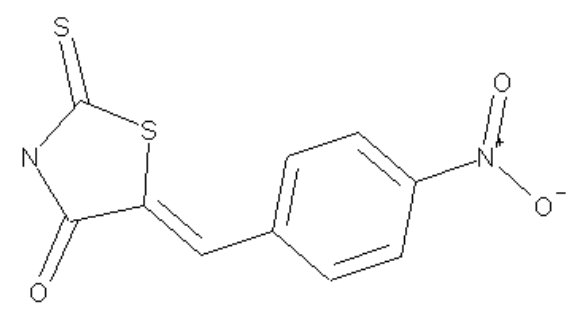

benzylidene

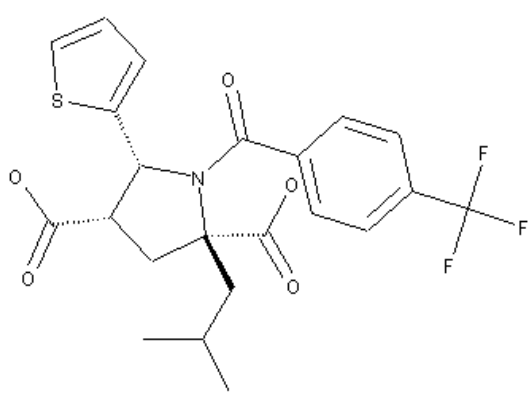

acyl pyrrolidine

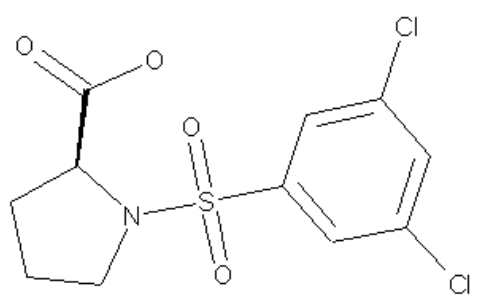

proline sulfonamide

Recently, Support Vector Machine (SVM) method has been used to build classification models for separating a series of compounds into inhibitors and non-inhibitors [11-13], or high active and low active ligands $[14,15]$ of a protein target to study the structure bioactivity relationship in drug design. The molecular descriptors used for building models can be fast calculated according to the structure of a molecule. So the SVM models could be used for further ligand-based virtual screening from a large compound library [13]. Using ligand-based SVM classification models, only a few potential inhibitors will be chosen for bioassay test. This methodology could reduce the time and cost for new drug discovery.

Lv et al. [16] built computational models using several machine learning (ML) methods (support vector machine (SVM), k-nearest neighbor $(\mathrm{k}-\mathrm{NN})$, and $\mathrm{C} 4.5$ decision tree (C4.5 DT)) for predicting NS5B polymerase inhibitors on a dataset of 1313 compounds, including 552 inhibitors $\left(\mathrm{IC}_{50}<400 \mathrm{nM}\right)$, 696 non-inhibitors $\left(\mathrm{IC}_{50}>600 \mathrm{nM}\right)$ and 65 compounds, whose activities range between inhibitors and non-inhibitors ( $400 \mathrm{nM}<\mathrm{IC}_{50}<600 \mathrm{nM}$ ). The prediction accuracy for their best model is up to $91.7 \%$ for NS5BIs and $78.2 \%$ for non-NS5BIs, which was built using a support vector machine (SVM). However, in their models, the HCV NS5B polymerase inhibitors which bind to the different binding sites were put together and were not distinguished.

In this study, a dataset containing 386 NNIs (non-nucleoside analogue inhibitors) fitting into the NNI III binding site of HCV NS5B polymerase, was complied. Each molecule was represented by molecular descriptors calculated from ADRIANA.Code [17]. Using a support vector machine (SVM), three classification models were built to predict whether a compound is active or weakly active as an inhibitor of NS5B polymerase based on a training set containing 266 compounds. And a test set containing 102 compounds was used to validate the models. 
Table 1. The intercorrelations between the 13 selected global descriptors and the activity ${ }^{\mathrm{a}}$.

\begin{tabular}{|c|c|c|c|c|c|c|c|c|c|c|c|c|c|}
\hline & Activity & InertiaZ & HAcc & NAtoms & NViolationsRo5 & $\log S$ & InertiaX & Span & HDon & HDon_N & NRotBond & RComplexity & Dipole \\
\hline InertiaZ & 0.540 & 1 & & & & & & & & & & & \\
\hline HAcc & 0.492 & 0.794 & 1 & & & & & & & & & & \\
\hline NAtoms & 0.457 & 0.812 & 0.841 & 1 & & & & & & & & & \\
\hline NViolationsRo5 & 0.452 & 0.770 & 0.734 & 0.607 & 1 & & & & & & & & \\
\hline $\log S$ & -0.439 & -0.735 & -0.404 & -0.607 & -0.475 & 1 & & & & & & & \\
\hline InertiaX & 0.426 & 0.830 & 0.720 & 0.822 & 0.673 & -0.721 & 1 & & & & & & \\
\hline Span & 0.403 & 0.824 & 0.677 & 0.760 & 0.501 & -0.625 & 0.667 & 1 & & & & & \\
\hline HDon & 0.397 & 0.629 & 0.724 & 0.655 & 0.515 & -0.241 & 0.569 & 0.523 & 1 & & & & \\
\hline HDon_N & 0.364 & 0.653 & 0.604 & 0.503 & 0.553 & -0.314 & 0.460 & 0.576 & 0.792 & 1 & & & \\
\hline NRotBond & 0.323 & 0.686 & 0.681 & 0.759 & 0.615 & -0.431 & 0.702 & 0.658 & 0.461 & 0.348 & 1 & & \\
\hline RComplexity & 0.298 & 0.280 & 0.426 & 0.497 & -0.007 & -0.246 & 0.266 & 0.483 & 0.366 & 0.259 & 0.077 & 1 & \\
\hline Dipole & 0.296 & 0.303 & 0.416 & 0.464 & 0.067 & -0.306 & 0.322 & 0.534 & 0.288 & 0.237 & 0.179 & 0.794 & 1 \\
\hline Eccentric & 0.168 & 0.275 & 0.139 & -0.039 & 0.189 & -0.018 & -0.241 & 0.267 & 0.089 & 0.374 & -0.045 & 0.009 & -0.008 \\
\hline
\end{tabular}

${ }^{\mathrm{a}}$ InertiaZ represents principal component of the inertia tensor in z-direction; HAcc represents number of hydrogen bonding acceptors derived from the sum of nitrogen and oxygen atoms in the molecule; NAtoms represents number of all atoms in the molecule; NViolationsRo5 represents number of violations of the Lipinski's rule of 5 (Weight $>500$, XlogP $>5$, HDon $>5$, HAcc > 10); LogS represents solubility of the molecule in water in [log units]; InertiaX represents principal component of the inertia tensor in $x$-direction; Span represents radius of the smallest sphere centered at the center of mass which completely encloses all atoms in the molecule; HDon represents number of hydrogen bonding donors derived from the sum of $\mathrm{N}-\mathrm{H}$ and O-H groups in the molecule; HDon_N represents number of hydrogen bonding donors N-H groups in the molecule; NRotBond represents number of open-chain, single rotatable bonds; RComplexity represents ring complexity according to the approach by J. Gasteiger and C. Jochum [18]; Dipole represents dipole moment in [Debye] of the molecule; Eccentric represents molecular eccentricity [19]. 


\section{Results and Discussion}

\subsection{Model 1 Built with Global Descriptors}

With the descriptor selection method (in Section 3.3), the 27 global descriptors were chosen. From them, 13 descriptors were selected. The 13 selected global descriptors and their correlations with the activity are shown in Table 1.

Then Model 1 was built with the 13 selected global descriptors using SVM. The training set including 266 compounds was used to generate the model, and the test set including 102 compounds was used to test the model. The two parameters of the $\operatorname{SVM}(c, g)$ were selected using the auto-searching program "grid.py" through a fivefold cross-validation in Libsvm. Afterwards, manual selection was done, and the optimum parameters of $c=0.00097656, g=8$ were selected to build an SVM model. Model 1 had a prediction accuracy of $87.97 \%$ on training set, a prediction accuracy of $78.43 \%$ and MCC value of 0.625 on test set.

\subsection{Model 2 with Global Descriptors and 2D Autocorrelation Descriptors}

With the descriptor selection method (in Section 3.3), the 27 global descriptors and 88 2D autocorrelation descriptors were chosen. From them, 16 descriptors were selected. The 16 selected global and 2D autocorrelation descriptors and their correlations with the activity are shown in Table 2.

Table 2. The correlation coefficients between the 16 selected global and $2 \mathrm{D}$ autocorrelation descriptors and the activity.

\begin{tabular}{|c|c|c|}
\hline & Activity & Description of Selected Descriptors \\
\hline Span & 0.403 & $\begin{array}{l}\text { Radius of the smallest sphere centered at the center of mass } \\
\text { which completely encloses all atoms in the molecule }\end{array}$ \\
\hline NRotBond & 0.323 & Number of open-chain, single rotatable bonds \\
\hline $\log S$ & -0.439 & Solubility of the molecule in water in [log units] \\
\hline InertiaZ & 0.540 & Principal component of the inertia tensor in $z$-direction \\
\hline InertiaX & 0.426 & Principal component of the inertia tensor in $x$-direction \\
\hline 2DACorr_TotChg_11 & -0.277 & $\begin{array}{l}\text { The eleventh component of 2D autocorrelation coefficients for } \\
\sigma \text { and } \pi \text { charges, where the distance } d=10\end{array}$ \\
\hline 2DACorr_TotChg_1 & 0.523 & $\begin{array}{l}\text { The first component of 2D autocorrelation coefficients for } \sigma \\
\text { and } \pi \text { charges, where the distance } d=0\end{array}$ \\
\hline 2DACorr_SigChg_4 & -0.452 & $\begin{array}{l}\text { The fourth component of 2D autocorrelation coefficients for } \sigma \\
\text { charge, where the distance } d=3\end{array}$ \\
\hline 2DACorr_SigChg_3 & 0.272 & $\begin{array}{l}\text { The third component of 2D autocorrelation coefficients for } \sigma \\
\text { charge, where the distance } d=2\end{array}$ \\
\hline 2DACorr_SigChg_2 & -0.249 & $\begin{array}{l}\text { The second component of 2D autocorrelation coefficients for } \sigma \\
\text { charge, where the distance } d=1\end{array}$ \\
\hline 2DACorr_PiChg_10 & 0.326 & $\begin{array}{l}\text { The tenth component of 2D autocorrelation coefficients for } \pi \\
\text { charges, where the distance } d=9\end{array}$ \\
\hline 2DACorr_LpEN_8 & 0.305 & $\begin{array}{l}\text { The eighth component of } 2 \mathrm{D} \text { autocorrelation coefficient for } \\
\text { lone pair electronegativities, where the distance } d=7\end{array}$ \\
\hline
\end{tabular}


Table 2. Cont.

\begin{tabular}{lcl}
\hline & Activity & Description of Selected Descriptors \\
\hline 2DACorr_LpEN_6 & 0.582 & $\begin{array}{l}\text { The sixth component of 2D autocorrelation coefficient for lone } \\
\text { pair electronegativities, where the distance } d=5\end{array}$ \\
2DACorr_LpEN_4 & 0.198 & $\begin{array}{l}\text { The fourth component of 2D autocorrelation coefficient for } \\
\text { lone pair electronegativities, where the distance } d=3 \\
\text { The tenth component of 2D autocorrelation coefficient for lone } \\
\text { pair electronegativities, where the distance } d=9 \\
\text { The eleventh component of 2D autocorrelation coefficient for } \\
\text { identity, where the distance } d=10\end{array}$ \\
\hline 2DACorr_Ident_11 & 0.421 & 0.166
\end{tabular}

Then Model 2 was built with the 16 selected global and 2D autocorrelation descriptors using SVM. The optimum parameters of $c=0.00097656, g=16$ were selected to build an SVM model. Model 2 had a prediction accuracy of $95.49 \%$ on training set, a prediction accuracy of $88.24 \%$ and MCC value of 0.789 on test set.

\subsection{Model 3 with Global Descriptors and 3D Autocorrelation Descriptors}

With the descriptor selection method (in Section 3.3), the 27 global descriptors and 96 3D autocorrelation descriptors were chosen. From them, 19 descriptors were selected. The 19 selected global and 3D autocorrelation descriptors and their correlations with the activity are shown in Table 3.

Table 3. The correlation coefficients between the selected 19 global and $3 \mathrm{D}$ autocorrelation descriptors and the activity.

\begin{tabular}{|c|c|c|}
\hline & Activity & Description of Selected Descriptors \\
\hline HDon & 0.397 & $\begin{array}{l}\text { Number of hydrogen bonding donors derived from the sum of } \\
\mathrm{N}-\mathrm{H} \text { and } \mathrm{O}-\mathrm{H} \text { groups in the molecule }\end{array}$ \\
\hline HAcc_N & 0.431 & $\begin{array}{l}\text { Number of hydrogen bonding acceptors derived from the } \\
\text { nitrogen atoms in the molecule }\end{array}$ \\
\hline HAcc_O & 0.417 & $\begin{array}{l}\text { Number of hydrogen bonding acceptors derived from the } \\
\text { oxygen atoms in the molecule }\end{array}$ \\
\hline $\log S$ & -0.439 & Solubility of the molecule in water in [log units] \\
\hline NRotBond & 0.323 & Number of open-chain, single rotatable bonds \\
\hline InertiaX & 0.426 & Principal component of the inertia tensor in $x$-direction \\
\hline InertiaZ & 0.540 & Principal component of the inertia tensor in $z$-direction \\
\hline Span & 0.403 & $\begin{array}{l}\text { Radius of the smallest sphere centered at the center of mass } \\
\text { which completely encloses all atoms in the molecule }\end{array}$ \\
\hline Eccentric & 0.168 & Molecular eccentricity [19] \\
\hline 3DACorr_SigChg_2 & -0.210 & $\begin{array}{l}\text { 3D autocorrelation weighted by } \sigma \text { atom charges, } \\
\text { where } d \text { is in the range of } 2-3 \AA\end{array}$ \\
\hline 3DACorr_SigChg_6 & -0.364 & $\begin{array}{l}\text { 3D autocorrelation weighted by } \sigma \text { atom charges, } \\
\text { where } d \text { is in the range of } 6-7 \AA\end{array}$ \\
\hline 3DACorr_SigChg_7 & 0.345 & $\begin{array}{l}\text { 3D autocorrelation weighted by } \sigma \text { atom charges, } \\
\text { where } d \text { is in the range of } 7-8 \AA\end{array}$ \\
\hline
\end{tabular}


Table 3. Cont.

\begin{tabular}{|c|c|c|}
\hline & Activity & Description of Selected Descriptors \\
\hline 3DACorr_PiChg_4 & -0.165 & $\begin{array}{l}\text { 3D autocorrelation weighted by } \pi \text { atom charges, } \\
\text { where } d \text { is in the range of } 4-5 \AA\end{array}$ \\
\hline 3DACorr_PiChg_10 & 0.166 & $\begin{array}{l}\text { 3D autocorrelation weighted by } \pi \text { atom charges, } \\
\text { where } d \text { is in the range of } 10-11 \AA\end{array}$ \\
\hline 3DACorr_TotChg_1 & -0.514 & $\begin{array}{l}\text { 3D autocorrelation weighted by total atom charges } \\
\text { (sum of } \sigma, \pi \text { charges), where } \mathrm{d} \text { is in the range of } 1-2 \AA\end{array}$ \\
\hline 3DACorr_TotChg_7 & 0.348 & $\begin{array}{l}\text { 3D autocorrelation weighted by total atom charges } \\
\text { (sum of } \sigma, \pi \text { charges), where } \mathrm{d} \text { is in the range of } 7-8 \AA\end{array}$ \\
\hline 3DACorr_PiEN_7 & 0.436 & $\begin{array}{l}\text { 3D autocorrelation weighted by } \pi \text { atom electronegativities, } \\
\text { where } \mathrm{d} \text { is in the range of } 7-8 \AA\end{array}$ \\
\hline 3DACorr_LpEN_5 & 0.413 & $\begin{array}{l}\text { 3D autocorrelation weighted by lone pair electronegativities, } \\
\text { where } d \text { is in the range of } 5-6 \AA\end{array}$ \\
\hline 3DACorr_LpEN_12 & 0.350 & $\begin{array}{l}\text { 3D autocorrelation weighted by lone pair electronegativities, } \\
\text { where } d \text { is in the range of } 12-13 \AA\end{array}$ \\
\hline
\end{tabular}

Then Model 3 was built with the 19 selected global and 3D autocorrelation descriptors using SVM. The optimum parameters of $c=0.00097656, g=8$ were selected to build an SVM model. Model 3 had a prediction accuracy of $95.11 \%$ on training set, a prediction accuracy of $81.37 \%$ and MCC value of 0.681 on test set. The results for Model 1,2 and 3 are shown in Table 4.

Table 4. Prediction performance of the three SVM models ${ }^{\mathrm{a}}$.

\begin{tabular}{|c|c|c|c|c|c|c|c|}
\hline \multirow{2}{*}{ Model } & \multirow{2}{*}{$\begin{array}{l}\text { Number of } \\
\text { Descriptors }\end{array}$} & \multirow{2}{*}{$\frac{\text { Number of Compounds }}{\text { Training Set/Test Set }}$} & \multirow{2}{*}{$\begin{array}{c}\text { Training Set } \\
\text { Accuracy }\end{array}$} & \multicolumn{4}{|c|}{ Test Set } \\
\hline & & & & $\mathrm{SE}^{\mathrm{b}}$ & $S P^{c}$ & Accuracy & MCC $^{d}$ \\
\hline Moc & 13 & $266 / 102$ & $87.97 \%$ & $97.92 \%$ & $61.11 \%$ & $78.43 \%$ & 0.625 \\
\hline Model 2 & 16 & $266 / 102$ & $95.49 \%$ & $100 \%$ & $77.78 \%$ & $88.24 \%$ & 0.789 \\
\hline Model 3 & 19 & $266 / 102$ & $95.11 \%$ & $100 \%$ & $64.81 \%$ & $81.37 \%$ & 0.681 \\
\hline
\end{tabular}

${ }^{\mathrm{a}}$ Model 1 represents the model built with 13 selected global descriptors as shown in Table 1. Model 2 represents the model built with 16 selected global and 2D autocorrelation descriptors as shown in Table 2. Model 3 represents the model built with 19 selected global and 3D autocorrelation descriptors as shown in Table $3 ;{ }^{\mathrm{b}} \mathrm{SE}$ (sensitivity) represents the prediction accuracy of the active inhibitors; ${ }^{\mathrm{c}} \mathrm{SP}$ (specificity) represents the prediction accuracy of the weakly active inhibitors; ${ }^{\mathrm{d}}$ MCC represents Matthews Correlation Coefficient.

\subsection{Relationship between the Selected Molecular Descriptors and Activity}

It was found that some molecular properties such as molecular shape descriptors (InertiaZ, InertiaX and Span), number of rotatable bonds (NRotBond) and water solubility (LogS) played significance roles in predicting models, which occurred in each of the three models. Rotatable bonds (NRotBond) can represent the flexibility of a molecule. It can make a difference in the interaction between the ligand and protein. Principal component of the inertia tensor (InertiaX and InertiaZ) of a molecule performed high relevant with the activity.

Moreover, hydrogen bonding donor descriptor HDon (representing number of hydrogen bonding donors derived from the sum of $\mathrm{N}-\mathrm{H}$ and $\mathrm{O}-\mathrm{H}$ groups in the molecule.) occurred in both the Model 1 
and Model 3, hydrogen bonding acceptor descriptor HAcc (representing number of hydrogen bonding acceptors derived from the sum of nitrogen and oxygen atoms in the molecule.) appeared in Model 1, HAcc_N (Number of hydrogen bonding acceptors derived from the nitrogen atoms in the molecule) and HAcc_O (Number of hydrogen bonding acceptors derived from the oxygen atoms in the molecule) appeared in Model 3. It indicated that the hydrogen-bonding interactions were important for stabilizing the ligand in the HCV NS5B polymerase active centre.

In addition, the 2D autocorrelation for atom charges $(\sigma-, \pi-$ and total charge) and atom electronegativities (lone pair electronegativities) appeared in Model 2 (as shown in Table 2); while, the $3 \mathrm{D}$ autocorrelation for atom charges $(\sigma-, \pi$ - and total charge) and atom electronegativities (lone pair electronegativities) existed in Model 3 (as shown in Table 3). They indicated that the atom charge and electronegativity related descriptors were also important for the interaction between the ligand and NS5B polymerase.

\subsection{External Test Set}

A dataset containing 80 newly synthesized compounds (which also bind to the NNI III binding site of HCV NS5B polymerase) was collected from recent literatures [8,20,21], which were not included in the training and test set. The dataset was used as an external test set, which contains 38 active inhibitors and 42 weakly active inhibitors of NS5B polymerase. The molecular structures can be obtained in the external.sdf in Supporting Information. Prediction performances of the three SVM models are shown in Table 5. Model 1 with 13 selected global descriptors, which gave the best prediction performance on the external test set, had the highest prediction accuracy $(\mathrm{Q})$ of $86.25 \%$ and Matthews correlation coefficient (MCC) of 0.732 . Both the Model 1 and 2 have SE $92.11 \%$, but their SP are low, which means more weakly inhibitors were wrongly predicted to be active ones by these models. Although Model 2 had higher prediction accuracy on test set, Model 1 showed better prediction accuracy and MCC on external test set than those of Model 2. The reason might be that the molecular structures in the external test set are very different with those in the training set. The training set contains benzothiadiazine analogues, acyl pyrrolidine, benzylidene and proline sulfonamide. The external test set contains benzothiadiazine analogues and acrylic acid derivatives.

Table 5. Prediction accuracy on the external test set with three models ${ }^{\mathrm{a}}$. The external test set contains 38 active inhibitors and 42 weakly active inhibitors of NS5B polymerase.

\begin{tabular}{ccccccc}
\hline Model & $\begin{array}{c}\text { Number of } \\
\text { Descriptors }\end{array}$ & $\begin{array}{c}\text { Number of } \\
\text { Compounds }\end{array}$ & SE $^{\mathbf{b}}$ & SP $^{\mathbf{c}}$ & Accuracy & MCC $^{\mathbf{d}}$ \\
\hline Model 1 & 13 & 80 & $92.11 \%$ & $80.95 \%$ & $86.25 \%$ & 0.732 \\
Model 2 & 16 & 80 & $92.11 \%$ & $69.05 \%$ & $80.00 \%$ & 0.623 \\
Model 3 & 19 & 80 & $65.79 \%$ & $54.76 \%$ & $60.00 \%$ & 0.206 \\
\hline
\end{tabular}

${ }^{a}$ Model 1 represents the model built with 13 selected global descriptors as shown in Table 1. Model 2 represents the model built with 16 selected global and 2D Autocorrelation descriptors as shown in Table 2. Model 3 represents the model built with 19 selected global and 3D Autocorrelation descriptors as shown in Table $3 ;{ }^{\mathrm{b}} \mathrm{SE}$ (sensitivity) represents the prediction accuracy of the active inhibitors; ${ }^{\mathrm{c}} \mathrm{SP}$ (specificity) represents the prediction accuracy of the weakly active inhibitors; ${ }^{\mathrm{d}} \mathrm{MCC}$ represents Matthews Correlation Coefficient. 
One can see that Model 3 showed similar prediction ability with that of Model 1 for the test set. However, compared with Model 1 and Model 2, Model 3 performed poorly on external test set. It was supposed that the molecular structures in external test set are very different with those in the training set. It was observed that acrylic acid derivatives in the external test set were not contained in the training set. It seemed that the 3D descriptors (for building Model 3) were more sensitive than global descriptors (for building Model 1) and 2D descriptors (for building Model 2). As for predicting the activity of the unknown molecules, Model 1 and Model 2 might be of preferred use.

\section{Experimental Section}

\subsection{Dataset}

All compounds used in this work were taken from references [2,22-31]. (All the compounds with their experimental $\mathrm{IC}_{50}$ values were contained in our datasets. The molecular structures of the compounds are shown in the Supporting Information). The $\mathrm{IC}_{50}$ values of the compounds are those for HCV genotype 1.

The dataset includes 386 HCV NS5B NNI III binding site inhibitors. The activity values of the inhibitors cover a broad range from 2 to $30,000 \mathrm{nM}$. We considered the compounds with $\mathrm{IC}_{50}<400 \mathrm{nM}$ as active and $\mathrm{IC}_{50}>600 \mathrm{nM}$ as weakly active [16]. The compounds whose activity (400 nm $\left.<\mathrm{IC}_{50}<600 \mathrm{nM}\right)$ are modest [16], were removed (18 compounds). Accordingly, 217 compounds are active and 151 compounds are weakly active ones. Herein "1" was assigned for active inhibitors and "0" was assigned for weakly active inhibitors. A completed listed of the compounds structures and their corresponding $\mathrm{IC}_{50}$ are shown in the file of supporting.sdf in Supporting Information.

We separated the dataset into a training set and a test set. The training/test set selection was done by clustering the compounds based on the fingerprint MACCS [32]. Compounds of similar structural and chemical features were evenly assigned into separate sets using Kohonen's self-organizing map (calculated with the SONNIA software) [33]. For those compounds without enough structurally and chemically similar counterparts, they were put into the training set. Training set included 266 compounds, which consisted of 169 active compounds and 97 weakly active compounds. Test set included 102 compounds, which consisted of 48 active compounds and 54 weakly active compounds.

Molecular structure building and energy minimization were carried out using the software MOE (Molecular Operation Environment) [32]. The need for computer-generated 3D molecular structures has been recognized in drug design and many other areas. In this work, the optimization of 3D molecular structure was generated by the software CORINA [34].

\subsection{Molecular Descriptors}

A total of 211 descriptors were calculated using ADRIANA.Code [10], including 27 global molecular descriptors (including 8 size and shape descriptors), 88 2D property autocorrelation descriptors and 96 3D property autocorrelation descriptors.

A global molecular descriptor represents each chemical structure by a structural, chemical or physicochemical feature or property of the molecule expressed by a single value. A size and shape 
descriptor represents a molecule by its 3D structure, and hydrogen atoms are taken into account. For example, the descriptor NRotBond can stand for number of open-chain, single rotatable bonds [35].

The 2D property autocorrelation uses the $2 \mathrm{D}$ molecular structure and atom pair properties as a basis to obtain vectorial molecular descriptors. And the atom pair properties are summed up for certain topological distances which count the number of bonds on the shortest path between two atoms. The 2D molecular autocorrelation vectors are calculated by the following equation (Equation (1)):

$$
A(d)=\frac{1}{2} \sum_{\substack{i, j \\ i \neq j}} p_{i} p_{j} \delta_{i j}\left(d-d_{i j}\right)
$$

$A(d)$ is the topological autocorrelation coefficient referring to atom pairs $i, j$ which are separated by $d$ bonds. $p_{i}, p_{j}$ is an atomic property such as partial atomic charge on atom $i$ or $j$, respectively. Thus, for each compound, a series of coefficients for different topological distances $d$, a so-called autocorrelation vector is obtained. The $2 \mathrm{D}$ molecular autocorrelation vectors were calculated based on the following eight atomic properties: $\sigma$ charge (SigChg), $\pi$ charge (PiChg), total charges (TotChg), $\sigma$ electronegativity (SigEN), $\pi$ electronegativity (PiEN), lone-pair electronegativity ( $\mathrm{ppEN}$ ), atomic polarizability (Apolariz) and identity (Ident). For each molecule, all the hydrogen atoms were excluded. For each property, the autocorrelation values for eleven distances ( $0-10$ bonds) were calculated. Thus, for each molecule, 88 2D property autocorrelations can be obtained.

Molecules are spatial objects in 3D space. Autocorrelation can also be applied to the 3D structure of a molecule. Thus, the resulting autocorrelation vectors do not only code for the spatial arrangement of the atoms but also for spatial distribution of physicochemical properties in a molecule. Since the distances $d$ and $d_{i j}$ (in Equation (1)) are continuous distances in 3D space between the atoms $i$ and $j$ (in $[\AA]$ ), an additional binning of $d$ into certain distance intervals (e.g., in steps of $1 \AA$ ) is necessary to transform the function $A(d)$ into a vector $A\left(d_{n}\right)$ of size $n$.

$$
A\left(d_{n}\right)=\frac{1}{2 L_{n}} \sum_{\substack{i, j \\ i \neq j}} p_{i} p_{j}
$$

In Equation (2), $L_{n}$ is the number of distances occurring in a certain distance interval and $p_{i}$ and $p_{j}$ are the atom properties of the atoms $i$ and $j$. The sampling of all distances in $n$ equidistant intervals (e.g., in distance bins of 1-2 $\AA, 2-3 \AA, 3-4 \AA$, ...) results in an n-dimensional vector of autocorrelation coefficients. The atom property $p$ used for the calculation of the $3 \mathrm{D}$ autocorrelation coefficients can either be simply the identity (Identity: $p_{i}=p_{j}=1$ ) or any physicochemical atom property, such as charge distributions or polarizability effects. (Here, eight atom properties as above were computed). For each molecule, all the hydrogen atoms were excluded. For each of the eight properties, a series of 12 vectors were computed, where $L_{n}$ correspond to the 12 3D distance intervals from 1-2 $\AA, 2-3 \AA, \ldots$ to $12-13 \AA$. Thus for each molecule, 96 3D properties autocorrelations can be obtained.

\subsection{Descriptors Selection}

Pearson correlation analysis [36] can reduce descriptors that are not significantly correlated with activity and highly correlated with each other. In this work, descriptors whose Pearson correlation coefficient with activity was less than 0.15 were deleted. If the pairwise correlation coefficient between 
any two descriptors was higher than 0.85 , the descriptor that had a lower correlation coefficient with the activity was removed. Then a stepwise variable selection method was carried out. All the descriptors selected by correlation analysis were sorted in a descending order according to their correlation coefficient with activity. Simple linear regression of activity and the first descriptor was done to obtain an initial equation. Other descriptors were then added to the regression equation one by one. A significance test was carried out for each new regression equation and every descriptor in the equation. If the new regression equation was not "statistically significant" because of the addition of a new descriptor, that the new descriptor was removed. Descriptors that are not "statistically significant" in the equation were also removed, followed by construction of a new regression equation. The process was continued until no descriptor can be added or removed by this approach. Before training, the input data (selected descriptors) were scaled to a $[0.1,0.9]$ range via the Equation (3).

$$
x_{i}^{*}=\frac{x_{i}-x_{\min }}{x_{\max }-x_{\min }} \times 0.8+0.1
$$

where $X_{i}$ was the original value, and $X_{\mathrm{i}}^{*}$ was the scaled value. $X_{\min }$ and $X_{\max }$ were the corresponding minimum and maximum values of the descriptor variable, respectively.

\subsection{Support Vector Machine}

The SVM [37] technique was applied to build the classification models of NS5B polymerase inhibitors. SVM is a useful tool for classification. It is based on the Vapnik Chervonenkis dimension and Vapnik's Structural Risk Minimization principle. Its main idea is to map data into a high-dimensional space in which a constrained quadratic programming problem will be solved and a separating hyperplane with the maximal margin will be found.

In this study, the Libsvm [38] program was utilized to build SVM models. Libsvm software is developed by Chang and Lin for SVM analysis. This software is based on the function of classification. There are four basic kernels in Libsvm software. The commonly used kernel, the Radial Basis Function (RBF) kernel (Equation (4)), was used. It is used to convert the data into a higherdimensional space. The parameters $c$ (Equation (5)) and g were chosen by the auto-searching program "'grid.py" through a cross-validation method.

$$
\begin{gathered}
\mathrm{k}(\mathrm{x}, \mathrm{y})=\exp (-\mathrm{g}\|\mathrm{x}-\mathrm{y}\| 2) \\
\min _{w, b, \xi} \frac{1}{2} W^{T} W+C \sum_{i=1}^{1} \xi_{i} \\
\text { Subject to } \quad y_{i}\left(W^{T} \phi\left(X_{i}\right)+b\right) \geq 1-\xi_{i} \\
\xi_{i} \geq 0
\end{gathered}
$$

\subsection{Evaluation of Models}

Several methods were employed to evaluate the performance of the classification models, such as sensitivity (SE), specificity (SP), the overall prediction accuracy (Q) and Matthews correlation coefficient (MCC), which are listed by Equations (6-9). True positives (TP) stands for the number of active inhibitors which were predicted as active inhibitors, true negatives (TN) stands for the number of weakly active inhibitors which were predicted as weakly active inhibitors, false positives (FP) 
stands for the number of weakly active inhibitors which were predicted as active inhibitors and false negatives (FN) stands for the number of active inhibitors which were predicted as weakly active inhibitors. SE stands for the prediction accuracy for active inhibitors and SP stands for the prediction accuracy for weakly active inhibitors, respectively.

$$
\begin{gathered}
S E=(T P /(T P+F N)) \times 100 \% \\
S P=(T N /(T N+F P)) \times 100 \% \\
Q=((T P+T N) /(T P+T N+F P+F N)) \times 100 \% \\
M C C=\frac{T P \times T N-F N \times F P}{\sqrt{(T P+F N)(T P+F P)(T N+F N)(T N+F P)}}
\end{gathered}
$$

According to Equation (9), a higher MCC value means a better prediction performance.

\section{Conclusions}

In this study, three SVM models for classifying hepatitis C virus (HCV) NS5B NNI III binding site inhibitors were developed. All the three models showed good prediction ability for the test set. As for predicting the activity of the unknown molecular structures, Model 1 and Model 2 might be of preferred use.

It was found that some molecular properties such as molecular shape descriptors (InertiaZ, InertiaX and Span), number of rotatable bonds (NRotBond), water solubility (LogS), and hydrogen bonding related descriptors performed important roles in the interactions between the ligand and NS5B polymerase. In addition, the atom charges and atom electronegativities related descriptors were also important.

\section{Acknowledgments}

This work was supported by the National Natural Science Foundation of China (20605003 and 20975011) and "Chemical Grid Project" of Beijing University of Chemical Technology. We thank Molecular Networks GmbH, Erlangen, Germany for making the programs ADRIANA.Code, Corina, and SONNIA available for our scientific work.

\section{Conflict of Interest}

The authors declare no conflict of interest.

\section{References}

1. Koch, U.; Attenni, B.; Malancona, S.; Colarusso, S.; Conte, I.; di Filippo, M.; Harper, S.; Pacini, B.; Giomini, C.; Thomas, S.; et al. 2-(2-Thienyl)-5,6-dihydroxy-4-carboxypyrimidines as inhibitors of the hepatitis C virus NS5B polymerase: Discovery, SAR, modeling, and mutagenesis. J. Med. Chem. 2006, 49, 1693-1705.

2. Tedesco, R.; Shaw, A.N.; Bambal, R.; Chai, D.; Concha, N.O.; Darcy, M.G.; Dhanak, D.; Fitch, D.M.; Gates, A.; Gerhardt, W.G.; et al. 3-(1,1-dioxo-2H-(1,2,4)-benzothiadiazin-3-yl)-4- 
hydroxy-2(1H)-quinolinones, potent inhibitors of hepatitis $\mathrm{C}$ virus RNA-dependent RNA polymerase. J. Med. Chem. 2006, 49, 971-983.

3. Ontoria, J.M.; Rydberg, E.H.; di Marco, S.; Tomei, L.; Attenni, B.; Malancona, S.; Hernando, J.I.M.; Gennari, N.; Koch, U.; Narjes, F.; et al. Identification and biological evaluation of a series of $1 H$-benzo[de]isoquinoline-1,3(2H)-diones as hepatitis $\mathrm{C}$ virus NS5B polymerase inhibitors. J. Med. Chem. 2009, 52, 5217-5227.

4. Chen, K.X.; Lesburg, C.A.; Vibulbhan, B.; Yang, W. A novel class of highly potent irreversible hepatitis C virus NS5B polymerase inhibitors. J. Med. Chem. 2012, 55, 2089-2101.

5. Krueger, A.C.; Madigan, D.L.; Jiang, W.W.; Kati, W.M.; Liu, D.; Liu, Y.; Maring, C.J.; Masse, S.; McDaniel, K.F.; Middleton, T.; et al. Inhibitors of HCV NS5B polymerase: Synthesis and structure-activity relationships of $\mathrm{N}$-alkyl-4-hydroxyquinolon-3-yl-benzothiadiazine sulfamides. Bioorg. Med. Chem. Lett. 2006, 16, 3367-3370.

6. Ishida, T.; Suzuki, T.; Hirashima, S.; Mizutani, K.; Yoshida, A.; Ando, I.; Ikeda, S.; Adachi, T.; Hashimoto, H. Benzimidazole inhibitors of hepatitis C virus NS5B polymerase: Identification of 2-[(4-diarylmethoxy)phenyl]-benzimidazole. Bioorg. Med. Chem. Lett. 2006, 16, 1859-1863.

7. Das, D.; Hong, J.; Chen, S.H.; Wang, G.; Beigelman, L.; Seiwert, S.D.; Buckman, B.O. Recent advances in drug discovery of benzothiadiazine and related analogs as HCV NS5B polymerase inhibitors. Bioorg. Med. Chem. 2011, 19, 4690-4703.

8. Pfefferkorn, J.A.; Greene, M.L.; Nugent, R.A.; Gross, R.J.; Mitchell, M.A.; Finzel, B.C.; Harris, M.S.; Wells, P.A.; Shelly, J.A.; Anstadt, R.A.; et al. Inhibitors of HCV NS5B polymerase. Part 1: Evaluation of the southern region of (2Z)-2-(benzoylamino)-3-(5-phenyl-2-furyl)acrylic acid. Bioorg. Med. Chem. Lett. 2005, 15, 2481-2486.

9. Donner, P.L.; Xie, Q.; Pratt, J.K.; Maring, C.J.; Kati, W.; Jiang, W.; Liu, Y.; Koev, G.; Masse, S.; Montgomery, D.; et al. Des-A-ring benzothiadiazines: Inhibitors of HCV genotype 1 NS5B RNA-dependent RNA polymerase. Bioorg. Med. Chem. Lett. 2008, 18, 2735-2738.

10. Powdrill, M.H.; Bernatchez, J.A.; Gotte, M. Inhibitors of the hepatitis C virus RNA-dependent RNA polymerase NS5B. Viruses 2010, 2, 2169-2195.

11. Wang, Z.; Chen, Y.; Liang, H.; Bender, A.; Glen, R.C.; Yan, A. P-glycoprotein substrate models using support vector machines based on a comprehensive data set. J. Chem. Inf. Model. 2011, 51, 1447-1456.

12. Subramaniam, S.; Mehrotra, M.; Gupta, D. Support vector machine based classification model for screening Plasmodium falciparum proliferation Inhibitors and non-inhibitors. Biomed. Eng. Comput. Biol. 2011, 3, 13-24.

13. Liew, C.Y.; Ma, X.H.; Liu, X.; Yap, C.W. SVM model for virtual screening of Lck inhibitors. J. Chem. Inf. Model. 2009, 49, 877-885.

14. Armutlu, P.; Ozdemir, M.E.; Uney-Yuksektepe, F.; Kavakli, I.H.; Turkay, M. Classification of drug molecules considering their $\mathrm{IC}_{50}$ values using mixed-integer linear programming based hyper-boxes method. BMC Bioinforma. 2008, 9, doi:10.1186/1471-2105-9-411.

15. Hao, M.; Li, Y.; Wang, Y.; Zhang, S. A classification study of respiratory syncytial virus (RSV) inhibitors by variable selection with random forest. Int. J. Mol. Sci. 2011, 12, 1259-1280.

16. Lv, W.; Xue, Y. Prediction of hepatitis c virus non-structural proteins 5B polymerase inhibitors using machine learning methods. Acta Phys. Chim. Sin. 2011, 27, 1407-1416. 
17. ADRIANA.Code, Molecular Networks GmbH, Erlangen, Germany. Available online: http://www.molecular-networks.com (accessed on 22 March 2012).

18. Gasteiger, J.; Jochum, C. An algorithm for the perception of synthetically important rings. J. Chem. Inf. Comput. Sci. 1979, 19, 43-48.

19. Todeschini, R.; Consonni, V. Handbook of Molecular Descriptors; Wiley-VCH: Weinheim, Germany, 2000; Volume 11.

20. Pfefferkorn, J.A.; Nugent, R.; Gross, R.J.; Greene, M.; Mitchell, M.A.; Reding, M.T.; Funk, L.A.; Anderson, R.; Wells, P.A.; Shelly, J.A.; et al. Inhibitors of HCV NS5B polymerase. Part 2: Evaluation of the northern region of (2Z)-2-benzoylamino-3-(4-phenoxy-phenyl)-acrylic acid. Bioorg. Med. Chem. Lett. 2005, 15, 2812-2818.

21. Zhou, Y.; Webber, S.E.; Murphy, D.E.; Li, L.S.; Dragovich, P.S.; Tran, C.V.; Sun, Z.; Ruebsam, F.; Shah, A.M.; Tsan, M.; et al. Novel HCV NS5B polymerase inhibitors derived from 4-(1',1'-dioxo1',4'-dihydro-1'lambda6-benzo[1',2',4']thiadiazin-3'-yl)-5-hydroxy-2H-pyridazin-3-ones. Part 1: Exploration of 7'-substitution of benzothiadiazine. Bioorg. Med. Chem. Lett. 2008, 18, 1413-1418.

22. Burton, G.; Ku, T.W.; Carr, T.J.; Kiesow, T.; Sarisky, R.T.; Lin-Goerke, J.; Baker, A.; Earnshaw, D.L.; Hofmann, G.A.; Keenan, R.M.; et al. Identification of small molecule inhibitors of the hepatitis $\mathrm{C}$ virus RNA-dependent RNA polymerase from a pyrrolidine combinatorial mixture. Bioorg. Med. Chem. Lett. 2005, 15, 1553-1556.

23. De Vicente, J.; Hendricks, R.T.; Smith, D.B.; Fell, J.B.; Fischer, J.; Spencer, S.R.; Stengel, P.J.; Mohr, P.; Robinson, J.E.; Blake, J.F.; et al. Non-nucleoside inhibitors of HCV polymerase NS5B. Part 2: Synthesis and structure-activity relationships of benzothiazine-substituted quinolinediones. Bioorg. Med. Chem. Lett. 2009, 19, 3642-3646.

24. De Vicente, J.; Hendricks, R.T.; Smith, D.B.; Fell, J.B.; Fischer, J.; Spencer, S.R.; Stengel, P.J.; Mohr, P.; Robinson, J.E.; Blake, J.F.; et al. Non-nucleoside inhibitors of HCV polymerase NS5B. Part 3: Synthesis and optimization studies of benzothiazine-substituted tetramic acids. Bioorg. Med. Chem. Lett. 2009, 19, 5648-5651.

25. Dragovich, P.S.; Blazel, J.K.; Ellis, D.A.; Han, Q.; Kamran, R.; Kissinger, C.R.; LeBrun, L.A.; Li, L.S.; Murphy, D.E.; Noble, M.; et al. Novel HCV NS5B polymerase inhibitors derived from 4-(1',1'-dioxo-1',4'-dihydro-1'lambda(6)-benzo[1',2',4'] thiadiazin-3'-yl)-5-hydroxy-2H-pyridazin3-ones. Part 5: Exploration of pyridazinones containing 6-amino-substituents. Bioorg. Med. Chem. Lett. 2008, 18, 5635-5639.

26. Gopalsamy, A.; Chopra, R.; Lim, K.; Ciszewski, G.; Shi, M.; Curran, K.J.; Sukits, S.F.; Svenson, K.; Bard, J.; Ellingboe, J.W.; et al. Discovery of proline sulfonamides as potent and selective hepatitis C virus NS5b polymerase inhibitors. Evidence for a new NS5b polymerase binding site. J. Med. Chem. 2006, 49, 3052-3055.

27. Li, L.S.; Zhou, Y.; Murphy, D.E.; Stankovic, N.; Zhao, J.; Dragovich, P.S.; Bertolini, T.; Sun, Z.; Ayida, B.; Tran, C.V.; et al. Novel HCV NS5B polymerase inhibitors derived from 4-(1',1'-dioxo1',4'-dihydro-1'lambda(6)-benzo[1',2',4']thiadiazin-3'-yl)-5-hydroxy-2H-pyridazin-3-ones. Part 3: Further optimization of the 2-, 6-, and 7'-substituents and initial pharmacokinetic assessments. Bioorg. Med. Chem. Lett. 2008, 18, 3446-3455. 
28. Powers, J.P.; Piper, D.E.; Li, Y.; Mayorga, V.; Anzola, J.; Chen, J.M.; Jaen, J.C.; Lee, G.; Liu, J.; Peterson, M.G.; et al. SAR and mode of action of novel non-nucleoside inhibitors of hepatitis $\mathrm{C}$ NS5b RNA polymerase. J. Med. Chem. 2006, 49, 1034-1046.

29. Ruebsam, F.; Tran, C.V.; Li, L.S.; Kim, S.H.; Xiang, A.X.; Zhou, Y.; Blazel, J.K.; Sun, Z.; Dragovich, P.S.; Zhao, J.; et al. 5,6-Dihydro-1H-pyridin-2-ones as potent inhibitors of HCV NS5B polymerase. Bioorg. Med. Chem. Lett. 2009, 19, 451-458.

30. Ruebsam, F.; Webber, S.E.; Tran, M.T.; Tran, C.V.; Murphy, D.E.; Zhao, J.; Dragovich, P.S.; Kim, S.H.; Li, L.S.; Zhou, Y.; et al. Pyrrolo[1,2-b]pyridazin-2-ones as potent inhibitors of HCV NS5B polymerase. Bioorg. Med. Chem. Lett. 2008, 18, 3616-3621.

31. Tedesco, R.; Chai, D.; Darcy, M.G.; Dhanak, D.; Fitch, D.M.; Gates, A.; Johnston, V.K.; Keenan, R.M.; Lin-Goerke, J.; Sarisky, R.T.; et al. Synthesis and biological activity of heteroaryl 3-(1,1-dioxo-2H-(1,2,4)-benzothiadizin-3-yl)-4-hydroxy-2(1H)-quinolinone derivatives as hepatitis C virus NS5B polymerase inhibitors. Bioorg. Med. Chem. Lett. 2009, 19, 4354-4358.

32. MOE (The Molecular Operating Environment), version 2010; Chemical Computing Group Inc.: Montreal, Canada, 2010.

33. SONNIA Software, version 4; Molecular Networks GmbH: Erlangen, Germany, 2010.

34. Sadowski, J.; Gasteiger, J. From atoms and bonds to three-dimensional atomic coordinates: Automatic model builders. J. Chem. Rev. 1993, 93, 2567-2581.

35. Veber, D.F.; Johnson, S.R.; Cheng, H.Y.; Smith, B.R.; Ward, K.W.; Kopple, K.D. Molecular properties that influence the oral bioavailability of drug candidates. J. Med. Chem. 2002, 45, 2615-2623.

36. Rodgers, J.L.; Nicewander, W.A. Thirteen ways to look at the correlation coefficient. Am. Stat. 1988, 42, 59-66.

37. Cortes, C.; Vapnik, V.N. Support-vector networks. Mach. Learn. 1995, 20, 273-297.

38. Chang, C.C.; Lin, C.J. LIBSVM: A library for support vector machine. 2001. Available online: http://www.csie.ntu.edu.tw/ cjlin/libsvm (accessed on 22 March 2012).

(C) 2012 by the authors; licensee MDPI, Basel, Switzerland. This article is an open access article distributed under the terms and conditions of the Creative Commons Attribution license (http://creativecommons.org/licenses/by/3.0/). 